\title{
Sine felle columba. Sources et formation d'une formule épigraphique
}

Robert Favreau

\section{Citer ce document / Cite this document :}

Favreau Robert. Sine felle columba. Sources et formation d'une formule épigraphique. In: Cahiers de civilisation médiévale, 32e année ( $\left.n^{\circ} 126\right)$, Avril-juin 1989. pp. 105-113;

doi : https://doi.org/10.3406/ccmed.1989.2431

https://www.persee.fr/doc/ccmed_0007-9731_1989_num_32_126_2431

Fichier pdf généré le 25/03/2019 


\title{
Résumé
}

La Bible mentionne plusieurs fois la colombe : symbole de la paix avec la fin du déluge, oiseau du sacrifice, figure de l'Esprit Saint, image de la simplicité.

Les premiers auteurs chrétiens de Tertullien à Isidore de Séville ont commenté ces figures de la colombe, en particulier celle de la simplicité, oiseau sans fiel, sine felle columba selon l'expression qu'on trouve chez Arator au début du Vle s. et que l'abbé et l'abbaye de Saint-Riquier ont peut-être transmise en Gaule au IXe s. Sur les premières tombes chrétiennes la colombe symbolise l'âme simple et pure qui va trouver paix et béatitude au ciel.

Du IXe au XIIle s. on peut relever 21 épitaphes qui utilisent le mot columba, toujours en fin de vers. La possibilité d'avoir une rime riche avec tumba, emploi qu'on trouve dès 930 , a entraîné une fortune particulière de la formule au temps de la vogue du vers léonin riche, c'est-à-dire au Xle et surtout aux XIle et XIIle s. La fréquence du schéma ...tumba... columba (18 ex.) ou ...sine felle columba (10 ex.) suppose l'existence et la circulation de modèles épigraphiques.

\begin{abstract}
The dove is mentioned several times in the Bible, as a symbol of peace after the Flood, as the bird of sacrifice, as a representation of the Holy Spirit, as an evocation of simplicity. The earliest Christian authors, from Tertullian to Isidore of Seville, commented upon these images of the dove. Special attention was given to the embodiment of simplicity through the bird without wickedness, sine felle columba according to the expression used by Arator at the beginning of the Vlth century. This concept was possibly brought into Gaul by the abbot and abbey of Saint-Riquier in the IXth century. On the earliest Christian tombes the dove symbolized the pure and simple soul who will accede to heavenly beatitude and peace. From the IXth to the XIIIth century, 21 epitaphs make use of the word columba, and always at the end of a verse. The possibility of making a particularly good rhyme with tumba, a formula to be found as soon as 930 , brought about a certain popularity during the time when the rich leonine verse was in fashion, that is to say during the XIth, but especially during the XIlth and the XIIIth centuries. The frequency of the schema ...tumba... columba (18 examples) or ...sine felle columba (10 examples) implies the existence and circulation of epigraphic models.
\end{abstract}




\section{*Robert FAVREAU}

\section{SINE FELLE COLUMBA}

\section{Sources et formation d'une formule épigraphique}

RÉSUMÉ

La Bible mentionne plusieurs fois la colombe : symbole de la paix avec la fin du déluge, oiseau du sacrifice, figure de l'Esprit Saint, image de la simplicité.

Les premiers auteurs chrétiens de Tertullien à Isidore de Séville ont commentè ces figures de la colombe, en particulier celle de la simplicité, oiseau sans fiel, sine felle columba selon l'expression qu'on trouve chez $\Lambda$ rator au début du $\mathrm{vI}^{\mathrm{e}} \mathrm{s}$. et que l'abbé et l'abbaye de Saint-Riquier ont peut-être transmise en Gaule au IX ${ }^{*}$. Sur les premières tombes chrétiennes la colombe symbolise l'àme simple et pure qui va trouver paix et béatitude au ciel.

Du IX au XIII's. on peut relever 21 épitaphes qui utilisent le mot columba, toujours en fin de vers. La possibilité d'avoir une rime riche avec lumba, emploi qu'on trouve dès 930 , a entraîné une fortune particulière de la formule au temps de la vogue du vers léonin riche, c'est-à-dire au XI' et surtout aux XII' et XIII ${ }^{\mathrm{e}} \mathrm{s}$. La fréquence du schéma ... lumba ... columba (18 ex.) ou ... sine felle columba (10 ex.) suppose l'existence et la circulation de modèles épigraphiques.

The dove is mentioned several times in the Bible, as a symbol of peace after the Flood, as the bird of sacrifice, as a representation of the Holy Spirit, as an evocation of simplicity. The earliest Christian authors, from Tertullian to Isidore of Seville, commented upon these images of the dove. Special attention was given to the embodiment of simplicity through the bird without wickedness, sine felle columba according to the expression used by Arator at the beginning of the VIth century. This concept was possibly brought into Gaul by the abbot and abbey of Saint-Riquier in the IXth century. On the earliest. Christian tombes the dove symbolized the pure and simple soul who will accede to heavenly beatitude and peace. From the IXth to the XIIIth century, 21 epitaphs make use of the word columba, and always at the end of a verse. The possibility of making a particularly good rhyme with tumba, a formula to be found as soon as 930 , brought about a certain popularity during the time when the rich leonine verse was in fashion, that is to say during the XIth, but especially during the XIIth and the XIIIth centuries. The frequency of the schema ...lumba...columba (18 examples) or ...sine felle columba (10 examples) implies the existence and circulation of epigraphic models.

Lorsqu'on ouvre la concordance des inscriptions médiévales établie par l'équipe poitevine chargée de l'inventaire des inscriptions médiévales françaises, l'attention est de suite attirée par les regroupements de certaines formules qui témoignent de l'existence de modèles épigraphiques, formulaires, sylloges, auteurs particulièrement en vogue chez ceux qui s'exercent à la prosodie classique latine. Parmi ces regroupements un des plus spectaculaires est celui qui concerne le mot columba, qui se trouve toujours placé en fin d'hexamètre, presque toujours avec la rime tumba à l'hémistiche, et en des combinaisons diverses évoquant la simplicité de la colombe - simplex velut una columba -, et son absence de fiel - sine felle colomba - qui ne peuvent s'expliquer par le simple hasard de l'inspiration poétique. On peut, dans ce cas comme dans un certain nombre d'autres semblables, retrouver les sources, la formation, la fortune d'une formule épigraphique. 
Dans la Bible la colombe a diverses significations. Elle est celle qui guide l'arche de Noë et indique la fin du déluge par un rameau d'olivier aux feuilles verdoyantes ${ }^{1}$. Associée généralement à la tourterelle la colombe est aussi l'oiseau du sacrifice, aussi bien dans les instructions de Dieu à Abraham que dans de nombreuses prescriptions du Lévitique, et dans les scènes de la présentation au temple et des vendeurs chassés du temple chez les évangélistes ${ }^{2}$. La bien aimée du Cantique des Cantiques est plusieurs fois qualifiée de colombe, amica mea, columba mea ${ }^{3}$. L'iconographie de l'Esprit Saint descendant, sous la forme d'une colombe, sur le Christ lors de son baptême par Jean dans le Jourdain, est familière à chacun ${ }^{4}$. Enfin Matthieu rapporte l'enseignement de Jésus à ses disciples en Galilée : "Voici donc que je vous envoie comme des moutons parmi les loups. Soyez donc prudents comme des serpents et simples comme des colombes" ${ }^{5}$.

Plusieurs de ces significations n'ont eu que peu de suites dans l'épigraphie. En tant qu'oiseau du sacrifice, la colombe a été associée à la table d'autel, et à l'eucharistie. Le rapprochement avec la colombe du baptême dans le Jourdain est à l'origine des colombes eucharistiques que mentionnent la Vie de saint Basile attribuée à Amphiloque, la notice du pape Silvestre au Liber pontificalis, la dédicace de la basilique des Saints-Gervais-et-Protais par Innocent $I^{\mathrm{er}}$ au $\mathrm{v}^{*} \mathrm{~s}$., et bien d'autres textes ultérieurs, mais il ne nous reste aucun monument de cet ordre ${ }^{6}$. L'épitaphe de Nicolas II, abbé de Vauclair de 1222 à 1227 , nous offre un exemple de référence à l'offrande en sacrifice de la tourterelle et de la colombe :

\section{QLEM TEGIT HAEC TUMBA, TLRTUR FUIT ATQLE COLUMBA?.}

L'exemple est d'ailleurs intéressant en ce qu'il nous offre un exemple de rime entre tumba et columba - vers léonin riche - et nous montre l'utilisation d'une formule courante au premier hémistiche, la composition laborieuse du second hémistiche pour renvoyer aux expressions bibliques du sacrifice. Saint Ambroise cite la bien-aimée du Cantique dans son De Virginitate, pour souligner en particulier la "simplicité de la colombe" ${ }^{8}$ que l'on va retrouver par ailleurs. La colombe de l'Esprit Saint est souvent représentée dans l'iconographie du baptême de Jésus, mais avec une épigraphie des plus simples, reduite par exemple à SPIRITUS SANCTLS sur les fonts baptismaux de Saint-Barthélemy de Liège ${ }^{9}$. Plus intéressante pour notre propos est l'inscription du linteau en bâtière à la façade occidentale de l'église auvergnate de Champeix, qui accompagne la représentation symbolique de la Trinité - la Main divine, l'Agneau, la Colombe :

\section{TRES TRINUM SIGNANT POLLEX PECCUS ATQUE COLUMBA ${ }^{10}$.}

Dans un effort maladroit de versification on a placé à nouveau le mot columba en fin d'hexamètre, précédé d'un alque qui a permis de compléter le dactyle du cinquième pied.

La colombe de l'arche de Noë portant le rameau d'olivier fut considérée par les chrétiens du II's. comme le symbole de la paix du royaume des cieux que l'on souhaitait aux fidèles défunts. Sur un marbre provenant d'un des cimetières de Rome et conservé au musée du Latran, une longue

1. Genèse. VIII, 8-12

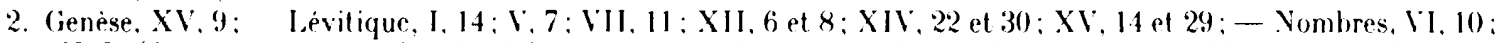
Luc: II, 24 (dans tous les exemples cités ici, lurlur el columba):-- pour les vendeurs du temple. Matthieu. XXI. 12:-Marc, XI, 15: - Jean, II. 14 et 16.

3. Cantique des cantiques, I, 14; II, 10, 13-14; IV. 1; V, 2, 12; VI, 8.

4. Matthieu, III, 16; - Marc, I, 10; - Jean I, 32.

5. Matthieu, X, 16. La Bible comporte encore plusieurs mentions de la colombe chez les prophetes, avec des sens divers qui n'ont pas eu la fortune des précédents.

6. H. Lfecterce, dans Diclionnaire d'archéologie chrétienne et de liturgie. III-2, Paris. 1914, col. 2231-2234.

7. Bibl. mun. Laon, ms. 85. feuille de garde à la fin du volume (Catalogue général des manuscrits des bibliotheques publiques de France. Paris, 1. I. 1849, 4", p. 86).

X. Lib. 1. rap. 12, dans P.I... XIV. col. 28.1.

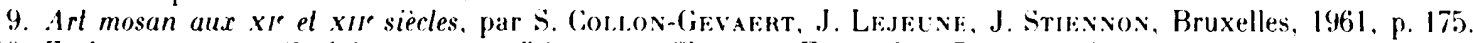

10. Z. Swifchowski, Sculpture romane d'Auwergne, Clemont-Ferrand, 1973, p. 34. fig. 14. 
épitaphe, Hic mihi semper dolor erit in aevo, etc., est accompagnée d'une colombe tenant dans son bec un rameau d'olivier à côté du mot : Pax. Dès la seconde moitié du II $^{\mathrm{r}} \mathrm{s}$. la colombe seule figure comme symbole chrétien de l'àme du fidèle dans la joie céleste. Cette figure, de création chrétienne, fut encore renforcée par la croyance ancienne en une représentation de l'âme humaine par un oiseau ${ }^{11}$. Cette croyance apparaît christianisée dans plusieurs récits anciens de martyres ${ }^{12}$, tels ceux de Polycarpe de Smyrne ${ }^{13}$ ou d'Eulalie de Merida ${ }^{14}$. La colombe va donc très tôt figurer sur les sarcohages, les épitaphes, et ce d'autant plus qu'on lui attribuera des qualités d'innocence, de pureté, de simplicité.

Le témoignage le plus ancien sur la colombe et sa signification chrétienne se trouve dans le De baptismo de Tertullien qui voit la nature de l'Esprit Saint signifiée par un animal de simplicité et d'innocence, sans fiel : la colombe. "C'est pourquoi, poursuit-il, le Christ a conseillé d'être simples comme des colombes. Après le déluge, continue-t-il aussitôt, l'antique iniquité a été purgée, il y a eu comme le baptème du monde, et la colombe a annoncé la paix de la céleste colère" ${ }^{15}$. Le passage est intéressant car apparaissent ici ramassés l'image de l'Esprit Saint figurée par la colombe, celle de la paix revenue dans le monde, qu'annonce la colombe de l'arche de Noë, et le conseil du Christ rapporté par Matthieu $(\mathrm{X}, 16)$. On y trouve le mot mème de simplicitatis, et un ipso felle careat columba d'où viendra l'expression sine felle. Evêque de Barcelone à la fin du IV's. saint Pacien compare l'Église de Dieu à une columbam non felle amaram ${ }^{16}$. Saint Ambroise déclare que l'Esprit est apparu au baptème du Christ sous la forme d'une colombe, à cause de la "simplicité" de celle-ci, et rappelle le conseil évangélique : soyez simples comme des colombes ${ }^{17}$. Augustin a composé un de ses sermons sur la "simplicité de la colombe" demandée à chaque chrétien (Matthieu, X, 16) ${ }^{18}$; dans un commentaire sur une lettre de Jean aux Parthes il voit dans la colombe la figure de la charité divine et ajoute : fel columba non habet ${ }^{19}$, dans un autre traite sur l'Evangile de Jean il insiste à plusieurs reprises sur la simplicité de la colombe : simplex, innocens, sine felle, pacata in osculis, non saeva in unguibus ${ }^{20}$. Dans une lettre au clergé et au peuple de Constantinople le pape Célestin $I^{\text {"r }}$ fait état de la "simplicité de la colombe" ${ }^{21}$ et sidoine Apollinaire fait de mème dans une lettre à Sulpice à la même date ${ }^{22}$. Cassiodore insiste sur l'innocence et la douceur de cet oiseau qui n'a aucune "volonté fielleuse" ${ }^{23}$. A deux reprises, commentant Matthieu, X, 16, Grégoire le (irand souligne la simplicité de la colombe, et il fait le rapprochement avec l'Esprit Saint qui s'est manifesté sous la forme d'une colombe ${ }^{24}$. Dans ses

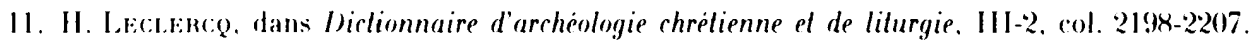

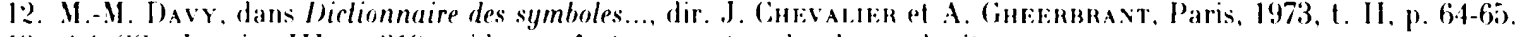

I:3. A.1 SS.. Janvier III, p. :319 : "id cum factum esset, columba fovolavit".

1.1. P.L.. L.X. col. 35): " "emicat inde columba repens".

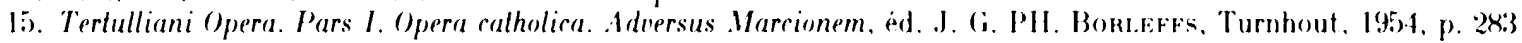
("Corpus christian. Ner. latina", 1).

16. P.I., XIII, col. 107X (epistola III, $\$ 21)$.

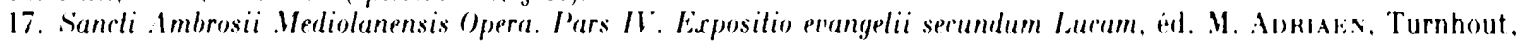

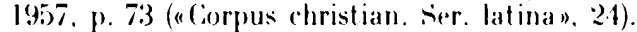

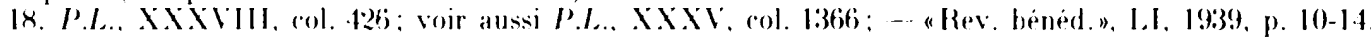

19. P.I., XXXV. col. 2035.

20. Sancti Aurelii Augustini, In Johannis evangelium tractatus CXXY\% ed. Radbotus Wir...Ms, o.s.B., Turnhout.

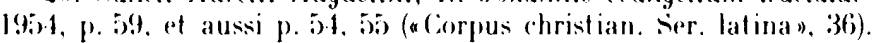

21. P.L... L. col. $18 \overline{\text {. }}$.

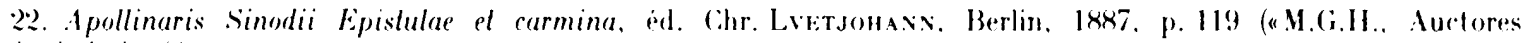
Antiquissimin", $x)$.

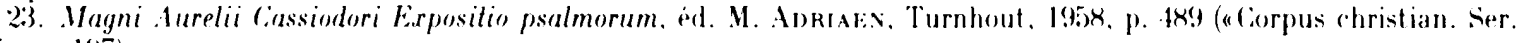
latina". 197).

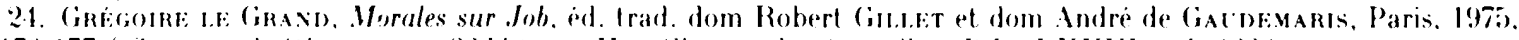
p. 176-177 ("sourees chretiennes", n"32bis): Homelies sur les evangiles, P.L.. I.XXVI, col. 1224. 
Elymologies Isidore de Séville reprend les mèmes qualités de la colombe, et notamment son absence de fiel, sine felle ${ }^{25}$.

Dans ces premiers siècles chrétiens, un certain nombre d'auteurs utilisent la métrique dans leurs œuvres. De l'évêque de . Vole, Paulin, nous retiendrons qu'il fait inscrire sur les portes latérales de la basilique Saint-Félix, des vers qui rappellent la simplicité de la colombe ${ }^{26}$. En cette première moitié du $v^{r}$ s. Coelius Sedulius met l'Évangile en vers et cite pour le baptème de Jésus, cet oiseau quae felle carel ${ }^{27}$. L'auteur le plus intéressant est ici le sous-diacre Arator qui présente au clergé de Rome, au début du vi" s., sa traduction en vers des Actes des apôtres, car on y trouve le vers :

Sufficit unda lavans, nisi sit sine felle columba ${ }^{28}$.

Venance Fortunat écrit à la fin du mème siècle :

\section{Qui sine felle manes in simplicitate columbae ${ }^{29}$}

L'expression même de sine felle columbas en fin de vers se retrouve même dans le traité De virginilate de l'Anglais Aldhelme, abbé de Malmesbury puis évêque de Sherborne à la fin du vir' et au début du viII" s. ${ }^{30}$.

Si l'on se tourne vers les premières inscriptions chrétiennes, on doit rappeler les fréquentes représentations de la colombe sur les épitaphes ${ }^{31}$, et les mentions de la colombe dans le texte mème des épitaphes. Il s'agit, dans ce dernier cas, d'inscriptions romaines. L'expression palumbus sine fel figure dans les épitaphes de Flavius Heliodorus et de Sabbatia, palumba sine fel dans celles de IDasuminia et d'Aselleca, palumbo sine felle dans celle de. Secunda, palumbulus sine fel in pace dans celle de Caccabonianus ${ }^{32}$. Mais la plus intéressante de ces épitaphes, pour notre propos, est une épitaphe trouvée à Rome, via Salaria, et qui nous est rapportée par la Sylloge de SaintRiquier éditée par J.-B. de Rossi33. On y trouve en effet, le vers :

\section{AIQUE SACERDOTEM SANCTUM, SINE FELLE COLUMBAM}

Nous savons que de nombreux papes ont été enterrés via Salaria, notamment au cimetière de Priscille, mais cette longue épitaphe ne comporte aucun nom. Elle a été attribuée par L. Duchesne au pape Libère, mort en septembre 356 et inhumé via Salaria dans le cimetière de Priscille ${ }^{34}$. Cette attribution a été reprise par 0. Marucchi ${ }^{35}$ qui fait observer que la longueur de la pièce, cinquante-quatre vers, l'écarte probablement des épitaphes réellement inscrites.

Ainsi du $I^{\circ}$ au viI" s. l'image de la simplicité, de la pureté, de l'innocence s'est attachée à la colombe, oiseau "sans fiel", et elle est devenue un des attributs de l'âme pure qui, détachée de ce

25. P.L., LXXXII, col. 467.

26. P.L. (Epistola XXXII), L.I, col. 337.

27. Sedulii opera omnia, ed. J. Iluemer, Vienne, 1885, p. 56 ("Corpus script. ecclesiast. latin." 10).

28. Aratoris subdiaconi, De actibus apostolorum, ed. Arthur Patch Mc KINıAY, Vienne, 1951 ("Corpus script. ecclesiast. latin." 72), livre I, vers 662 .

29. Venanti Honori Clementiani Fortunati presbiteri ilalici, Opera poetica, éd. Fr. Lro, Berlin, 1881, p. 51 ("M.(B.H., Auctores antiquissimi", IV/1).

30. Aldhelmi opera, éd. Rudolfus Ehwal. Berlin, 1919, p. 373 ("M.G.H., Auctores antiquissimi”, 15).

31. Pour le Recueil des inscriptions chréliennes de la Gaule antérieures à la Renaissance carolingienne, on peut voir de Nancy Gauthifr, I, Premiëre Belgique, Paris, 1975, p. 50-56, et Françoise Descombes, XV, Viennoise du Nord, Paris, 1985, p. 77.

32. E. Diғні, Inscriptiones latinae christianae veteres, I, Berlin, 1925, n"1561-1564, p. 299-300.

33. Inscriptiones christianae urbis Romae, vol. II, pars prima. Rome, 1888, p. 83, n"26:-Carmina latina epigraphica, ed. Fr. Buechatrer. Leipzig. 1895, 1. p. 373, n"787.

34. Le Liber ponlificalis, éd. L. I) uchessf, Paris, t. I, 1955, p. 208-210. L’éditeur écrit à propos de cette épitaphe qu'«il y a de graves raisons de la rapporter à ce pape".

35. O. Martcchi, Epigrafia cristiana .... Milan, 1910, n”445. p. 406-408. 
monde terrestre, va trouver paix et béatitude dans le royaume des cieux. L'expression sine felle s'est généralisée, le mot columba a remplacé celui de palumbus, et est apparue, en fin de vers, la formule sine felle columba. Qu'elle vienne de l'épitaphe du pape Libère au IV"s., du sous-diacre romain Arator au début du vi"s., qu'elle ait été répandue par l'intermédiaire de ces sylloges épigraphiques qui, telle la sylloge de Saint-Riquier, ont puisé avec prédilection dans les épitaphes romaines, ou par le succès de la version métrique des Actes des apôtres écrite par Arator, un point est sûr, c'est son origine romaine entre la seconde moitié du $\mathrm{IV}^{\circ}$ et le début du vir $\mathrm{s}$.

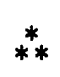

Les auteurs chrétiens du IX" au XII" s. continuent à souligner, en prose et en vers, l'innocence et la simplicité de la colombe. Theofridus d'Epternach dans sa Vie métrique de Willibrord écrit : Fugit ut accipitrem sine felle columba rapacem ${ }^{36}$, Milon de Saint-Amand dans sa Vie de saint Amand cite aussi les dulces sine felle columbas ${ }^{37}$, et les Carmina Cenlulensia font l'éloge de la simplicis ac retinens animum sine felle columbae ${ }^{38}$. Gautier de Liège vante à son tour la colombe Longius abjecta nigredine fellis amar ${ }^{39}$. Flodoard dans son éloge des saints Simon et Jude parle de l'astu serpentis cum simplicitate columbae ${ }^{40}$. Les louanges en vers adressées à l'archevêque de Cantorbéry Dunstan (959-988) rappellent le conseil transmis par Matthieu, X, 16 en une heureuse formule que l'on retrouvera en épigraphie,

Tu coluber prudens, simplex sine felle columba ${ }^{41}$.

Raoul Ardent et Geoffroi d'Admont composent des homélies sur le verset 16 du dixième chapitre de Matthieu, où l'on retrouve les mêmes mentions de simplicité (simplices, simplicilas) et d'innocence (sine felle, a malitia fellis aliena) ${ }^{42}$, Rupert de Deutz et Hugues de Saint-Victor énumèrent les sept ou dix propriétés de la colombe et relèvent d'entrée qu'elle est sine felle, felle caret $^{43}$. On trouve même l'expression dans les hymnes : $O$ columba sine felle $e^{44}$. Mais désormais l'image de la simplicité et de l'innocence de la colombe est pleinement entrée dans le domaine de l'épigraphie.

Les épitaphes qui font appel à la figure de la colombe évoquent souvent sa simplicité. On peut en présenter une douzaine d'exemples entre le $\mathrm{IX}^{\circ}$ et le XIII $\mathrm{s}^{45}$ auxquels on joindra l'épitaphe d'un certain Boson, à lzerche, au XI' s., où le mot même de columba n'est pas retenu, mais où la citation de Matthieu, X, 16, est présentée dans un distique:

\section{CUMQUE COLUMBINAM SERVARET SIMPLICITATEM SERPENTIS TAMEN INIULERAT SIBI CALLIDITATEM ${ }^{46}$,}

36. Vila Willibrordi metrica, èd. K. Rossbera, Leipzig, 1883, p. 54.

37. Poelae lalini aevi carolini, III, éd. L. Trat bF, Berlin. 1896, p. 581 ("M.G.H.. Poelarum latinorum Medii Aevi”, 3). 38. Ibid., p. 312, épitaphe datée par l'éditeur de 844-846?

39. Die lateinischen Dichter des deutschen . Mitlelalters. V. Die Ottonenzeil, éd. K. Strecker, N. Fickramans, G. Sulagi,

B. Brschoff, Leipig/Berlin/Munich, 1937-1979, p. 46.

40. P.L., CXXXV, col. $500^{\mathrm{B}}$.

41. Memoriales of Saint Dunslan Archbischop of Canlerbury, ed. William StvBbs, Londres, 1874, p. 371 ("Rerum britannic. medii aevi script.").

42. P.L., C.L.V, col. 1511 (R. Ardent): - P.L.. CI.XXIV. col. 860) (G. d'Admont).

43. P.L., CL.XVIII, col. 884, commentaire de Rupert sur le Cantique des Cantiques; P.L., CLXXXVII, col. 177, col. 19 et 20 , De bestiis d'Hugues de Saint-Victor.

44. Joseph Szöverffy, Marianische Molivik der Iymnen, Leyde, 1985. p. 110.

45. Voir le tableau des textes épigraphiques comportant le mot columba.

46. Corpus des inscriptions de la France médiévale [4]. Limousin, èd. R. Favrand et J. Michaud, Poitiers, 1978, p. 59. 
sous une forme que l'on peut rencontrer aussi dans la prose, ainsi dans l'Historia Sancli Florentii Salmurensis où le chroniqueur parlant de la mort de l'abbé sigon en 1070 le dit columbina simplicitale praedilus ${ }^{4}$.

Toutefois la fortune de l'emploi du mot columba à cette époque est certainement à mettre en relation avec la vogue du vers léonin riche, comportant rime par deux syllabes entre le premier et le second hémistiche. Dans une epitaphe le mot columba rimait parfaitement avec lumba, qui convenait à la fin du premier hémistiche dès lors qu'on l'employait à l'ablatif. On pouvait là utiliser des formules qui seront fort usuelles, hic jacet in lumba, hac jacet in tumba, clauditur hac tumba, qui jacet hac lumba ou toute autre variante. I)ans les 21 épitaphes données en annexe le mot columba est toujours à la fin du vers, le mot lumba est 17 fois à l'hémistiche, et une 18 fois se retrouve dans un distique où le premier hémistiche rime avec le dernier du second vers -... et le dernier du premier vers avec le premier du second - en 1275) au Vont-Saint-Éloi. On peut aussi écarter l'un des exemples, celui du $I x^{\prime}$ s., car le vers léonin riche n'est pas alors pratiqué. On arrive ainsi à 18 cas sur 20 entre 930 et 1305, ce qui suppose l'emploi de formulaires. Cette construction en ...lumba ...columba gardera toute sa faveur pendant tout le xil" s. alors que sera passée la grande période du vers léonin riche.

Dès lors qu'on avait les deux mots-clés de lumba et columba le complément du vers pouvait connaître de nombreux aménagements. On avait toutefois, tant chez les exégètes que les poètes, et encore dans l'épitaphe du pape libère et dans d'autres inscriptions chrètiennes de Rome l'expression sine felle, et mème son agencement en fin de vers, sine felle columba. Ce n'est pas une surprise de trouver l'expression chez . Vicon de Saint-Riquier au milieu du ix" s., puisque la sylloge connue sous le nom de Corbie ou de Saint-Riquier, qui contenait l'épitaphe du pape Libère a été écrite à la fin du viII" ou au début du $1 x^{\prime \prime}$ s. et se trouvait alors à Saint-Riquier. Avec l'épitaphe de maitre Hucbald, au $\mathrm{x}^{r}$ s., on reste dans la même région, et l'expression va ensuite se répandre largement puisqu'on la trouvera aussi bien dans la France méridionale que dans les Asturies ou en Allemagne, pour autant qu'on puisse faire un certain relevé en l'absence d'un corpus général. La formule sine felle columba est employée dans 10 des 21 exemples recueillis. Pour avoir un second hémistiche complet il fallait encore un mot de deux ou trois syllabes, et on aura ainsi quatre exemples de la formule simplex sine felle columba, qui a des parentés aver simplex velut una columba de l'abbaye d'Andres en 1274 et simplex velut ipse columba à Carpentras, en 1282, voire avec le simplex velut est sine felle columba du rouleau de l'abbé Girard, qui abandonna le vers léonin riche.

Si l'on veut conclure, on peut retenir quelques grands traits à propos des sources et de la diffusion des formules épigraphiques utilisant l'image de la colombe.

D'abord cette image de simplicité et d'innocence symbolisée par la colombe est issue des commentaires chrétiens des premiers siècles sur les textes bibliques. Il s'agit d'une création "chrétienne".

Des diverses figures pouvant être représentées par la colombe, c'est celle de l'âme pacifiée du défunt qui est presque exclusivement retenue dans l'épigraphie. Les expressions utilisant la comparaison avec la colombe se rattachent essentiellement au domaine des épitaphes.

L'utilisation du mot columba en finale d'hexamètre, précédé de la formule sine felle, est d'origine romaine, que l'on considère l'épitaphe qui pourrait être du pape Libère au IV" s., ou l'ceuvre du sous-diacre romain Arator au début du vi"s.

47. Chroniques des églises d'Anjou, éd. P. Marcheciay ef E. Mabnlok. Paris, 1869, p. 296. 
La transmission de la formule en Gaule a pu se faire par l'intermédiaire de l'abbaye de SaintRiquier. L'abbé Angilbert, mort en 814, est allé quatre fois à Rome, et c'est sans doute sous son abbatiat qu'ont été recueillies un certain nombre d'inscriptions romaines, dont l'épitaphe attribuée au pape Libère, dans une sylloge qui sera à partir du $\mathrm{xul}^{*} \mathrm{~s}$. dans l'abbaye de Corbie et que pour cette raison on appelle la sylloge de Saint-Riquier ou de Corbie ${ }^{48}$.

On peut ajouter que la bibliothèque de l'abbaye de Saint-Riquier, telle qu'Hariulf nous en donne l'état sous le règne de Louis le Pieux, comportait des ceuvres d'Augustin, de Grégoire, d'Isidore de Séville où figurent des commentaires sur la colombe, et encore de Sedulius et d'Arator ${ }^{49}$. C'est avec Micon diacre de Saint-Riquier à cette époque, que l'on retrouve l'expression sine felle columba en finale d'hexamètre.

La vogue des formules citant la colombe est en lien étroit avec la grande période du vers léonin riche et la commodité d'utiliser dans une épitaphe la rime riche ...lumba ...columba. Bien que cette étude n'envisage pas les textes des $\mathrm{XIV}^{\prime \prime}$ et $\mathrm{xV}^{\prime \prime}$ s., on peut penser que ces formules avec le mot "colombe" y sont beaucoup plus rares, du fait de la fin de l'emploi courant du vers léonin riche.

Enfin la généralisation de l'emploi des expressions simplex sine felle columba, ou seulement sine felle columba, laisse supposer l'existence de formulaires épigraphiques, el ce d'autant plus que d'autres formules très courantes sont employées dans le premier hémistiche. Sans doute les expressions ont pu continuer à ètre empruntées au tombeau romain, à quelque sylloge épigraphique, à l'œuvre d'Arator, etc., mais on voit mal que ces différentes sources puissent expliquer seules la généralisation des formules.

*Robert Favreal

C.E.S.C.M.

24 , rue de la Chaine

$\mathrm{F}-86022$ POITIERS

48. II. Lacifrce, dans Dirtionnaire d'archéologie chrélienne et de liturgie. VII-1, Paris, 1926, col. 882-88:3 (svlloges épigraphiques).

49. IAarict. Chronique de l'abbaye de Saint-Riquier ( $v$ s.-1104), éd. F. Lot. Paris, 1894, p. 89-92 ("Coll. textes pour servir a l'et. et à l'enseign. de l'hist."n). 


\section{ANNEXE}

\section{SIMPLICIS AC RETINENS ANIMUM SINE FELLE COLLMBAE}

Milon de Saint-Riquier, 844-846 ${ }^{1}$.

DORMIT IN HAC TUMBA, SIMPLEX SINE FELLE COLUMBA

Épitaphe d'Hucbald, $930^{2}$.

HIC JACET IN TUMBA, PRUDENS SIMPLEXQUE COLUMBA

Épitaphe de l'abbé Aimard, mort en $964^{3}$.

CLAUDITUR HAC TUMBA PRIDEM SINE FELLE COLUMBA

Épitaphe du comte Évrard, par Thierry, évèque de Metz, $978^{4}$.

ECCE SUB HAC TUMBA TEGITUR SINE FELLE COLUMBA

Épitaphe d'Anfroi, abbé des Préaux, mort en $1078^{5}$.

CLAUDITUR HAC TUMBA SIMPLEX SINE FELLE COLUMBA

Épitaphe de Frédéric, évêque de Liège, mort en $1127^{6}$.

FIT CINIS HAC TLMBA QUI SERPENS ATQUE COLUMBA

Epitaphe de Boson, abbé du Bec-Hellouin, $1136^{7}$.

NAMQUE FUIT SIMPLEX VELUT EST SINE FELLE COLUMBA

Rouleau d'un abbé Girard, première moitié $\mathrm{XII}^{\prime} \mathrm{s}{ }^{8}$.

SERPENS CAUTELA PLUS SIMPLICITATE COLUMBA

Epitaphe de l'abbé Wolfhelm, xı" s., Saint-Maximin de Trèves?.

CLALDITUR HAC TLMBA, JOB PECTORE MENTE COLUMBA

Épitaphe de Géraud de Jonquières, Toulouse, 1200 ou $1204^{10}$.

QUEM TEGIT HAEC TUMBA FLIT IN SERPENTE COLLMBA

Epitaphe de Robert, abbé de Saint-Nicolas d'Hermières, $1227^{11}$.

QUEM TEGIT HAEC TLMBA, TURTUR FUIT ATQLE COLUMBA

Epitaphe de Nicolas II, abbé de Vauclair, $1227^{12}$.

1. Poetae latini aevi carolini, III. éd. L. Tratbe, Berlin, 1896, p. 312 ("M.G.II., Poetarum latinorum Medii Aevi», 3).

2. P.L., CXXXII, col. 1049-1050; - Misloire littéraire de la France, t. VI, Paris, 1867, p. 213.

3. Conservée au musée de Cluny (Saòne-et-Loire) : note de M. Warisse "Bull. et Mém. Soc. archéol. et histor. de la Charente", 6" s., III. 1893, p. xxx-xxxı. Pensox, Cluny la ville et l'abbaye, $2 "$ éd., Cluny, 1884, p. 140.

4. P.L., CXXXVII, col. $364 ;-$ Poetae latini medii aevi. Die lateinischen Dichter des deutschen Mittelalters, $t$. V. Die

Ottonzeit, par K. Strecker. N. Fickfrmanx. (i. Silaci, B. Bischoff, Leipzig/Berlin/Munich, 1937/79, p. 309.

5. The Ecclesiastical History of Orderic Vitalis, IV, ed. Marjorie (iнınal.., Oxford, 1973, p. 304. p. 97.

6. Aegidii Aureavallensis, Gesta episcoporum Leodiensium, èd. I. HEt.tik, M.G.H., Scriptores, XXV, Hanovre, 1880,

7. L. Ifil.ssl., Notice sur vingl manuscrits du Valican. $\S X X$. Chronique du Bec n" 499 du Fonds de la Reine, "Bibl. Ec. des chartes", $8^{\prime \prime}$ s., II, 1876, p. 521-527.

8. Rouleaux des morts du IX' au Xve siècle, èd. L. Destast.e, Paris, 1866, p. 352

9. F. X. Kraus. Die christlichen Inschriften der Rheinlande, II. I)ie christlichen Inschriften von der mitte des achten bis zur mitte des dreizehnten Jahrhunderts, Fribourg-en-Brisgau/Leipzig, 1894, n"396. p. 192.

10. Corpus des inscriptions de la France médiénale. 7. Ville de Toulouse, par R. Favread, J. Michact, B. Leplant, Paris, 1982, n" 65, p. 106.

11. Gallia christiana. VII, Paris. 1744. col. 940-941.

12. Laon. Bibliothéque municipale. ms. 85. feuille de garde. 


\section{PAUSAT IN HAC TUMBA VIR SIMPLICITATE COLUMBA}

Épitaphe de Villiers-en-Brabant, $1^{\text {re }}$ moitié XıII' ${ }^{r} .^{13}$.

\section{HAC JACET IN TUMBA SIMPLEX SINE FELLE COLUMBA}

Épitaphe de Jourdain de Rabastens, Saint-Michel de Rabastens, $1264^{14}$.

HIC JACET IN TUMBA SIMPLEX VELUT UNA COLLMBA

Épitaphe de Boidin de Balinghem, provenant d'Andres, $1274^{15}$.

QUEM TEGIT HAC TUMBA MULTIS CARUS SIBI VILIS

VIR SAPIENS HUMILIS FUIT ET SINE FELLE COLUMBA

Épitaphe de l'abbé de Mont-Saint-Éloi, Jean de Barastre, $1275^{16}$.

ALBARLS HAC TUMBA JACET ACTU CORDE COLLMBA

Épitaphe d'Albarus à Córias (Asturies), $1276^{17}$.

QUI JACET HAC TUMBA SIMPLEX VELUT IPSE COLUMBA

Épitaphe de Raymond, prévôt de l'église de Carpentras, $1282^{18}$.

SUBJACET HUIC TUMBAE PAR SIMPLICITATE COLUMBAE

Épitaphe d'Arnoul de Romainville, abbé de Sainte-Geneviève de Paris, $1286^{19}$.

DORMIT IN HAC TUMBA SIMPLEX SINE FELLE COLUMBA

Epitaphe d'Étienne, chanoine de Saint-Ruf, $\mathrm{x} \mathrm{I}^{\mathrm{r}} \mathrm{s} .{ }^{20}$.

AICH JACET IN TUMBA SINE FELLE COLUMBA

Epitaphe de sœur Hailwige à Steinheim a.d. Murr, $1305^{21}$.

13. V. Huyghebafre, o.s.B., Arnoul ou Abond? A propos d'une épilaphe de Villiers-en-Brabanl, "Citeaux", XXXIII, 1982 , p. $392-396$.

14. Corpus des inscriplions de la France médievale. 9. Avegron, Lot, Tarn, par R. Favreau, J. Michaud, B. Lepiant, Paris, 1984, p. 140.

15. Epigraphie du département du Pas-de-Calais, publ. par la Commission départ. des Monuments historiques, Arras,

t. III, 1911, p. 51-52; pl. 3, pl. 308 (épitaphe conservée au Musée de Boulogne-sur-Mer).

16. Ibid., VIII, 1932, p. 331.

17. Ciriaco Miguel Vicill., Asturias monumental, epigrafica y diplomatica ..., Oviedo, 1887, p. 318

18. E. Andreoli et B.S. Lambert, Monographie de l'église calhédrale Saint-Siffrein de Carpeniras, Paris, 1862, p. 55.

19. Gallia christiana, VII, Paris, 1744, col. 745.

20. Vicente Garcia Loво, Las inscripciones de San Miguel de Fscalada. Estudio critico, Barcelone, 1982, n"24, p. 80.

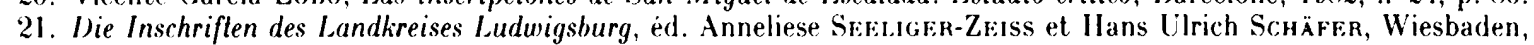
1986, n"21, p. 20-21 ("Die deutschen Inschriften", 25. "Heidelbergae Reihe", 9). 